\title{
Novel schemes for Compact FELs in the THz region: ENEA Experience and Perspectives
}

\author{
A. Doria, G. P. Gallerano \\ ENEA- Fusion Physics Division - Radiation Sources, Antennas and Diagnostics Laboratory \\ Via E. Fermi 45, 00044 Frascati - Italy, andrea.doria@enea.it
}

The rapid advance of Terahertz technologies, in terms of radiation generators, systems and scientific or industrial applications, have put a particular focus on compact sources with challenging performances in terms of generated power (peak and/or average), radiation time structure and frequency tunability.

Free Electrons based sources are probably the best candidates to express such a versatility; there are a number of schemes that have been investigated over the years to generate coherent radiation from free electrons in the mm-wave and Terahertz regions of the spectrum, covering a wide frequency range from approximately $100 \mathrm{GHz}$ to $10 \mathrm{THz}$. At such long wavelengths, good performance in terms of output power and gain can be achieved with a short length of the interaction region $(<50 \mathrm{~cm})$ and with different mechanisms of energy transfer from the electron beam to the radiation field. These include the magnetic undulator, dielectric loaded waveguides for Cerenkov emission, and metal grating devices, based on the Smith-Purcell effect. This study will proposes novel schemes for exploring the limits in performance of radio-frequency driven free electron devices in terms of ultra-short pulse duration, wide bandwidth operation and energy recovery for near $\mathrm{CW}$ operation.

\section{Auston Switch and Optical rectification}

Conventional $\mathrm{THz}$ emitters have gained popularity due to their characteristics and peculiarities. The so called Auston Switch, named after its inventor, and the Optical Rectifiers are the most widely used devices [1]. The Switch is made up with a coplanar strip antenna deposited on a non absorbing substrate. Along the strips there is a point where the gap is very small, of the order of $10 \mu \mathrm{m}$. If a powerful short laser pulse is focalised on this gap, the photons generate free carriers that drift under the action of the electric field generated by this bias voltage. This transient current gives rise to a time dependent dipole moment that acts as a source of a time dependent electric field according to expression. The result is the generation of an electric pulse which duration, and thus spectrum are related to the duration of the laser pulse. A picosecond or a sub-picosecond laser pulse generate a radiation burst in the $\mathrm{THz}$ range that is propagated and irradiated by the antenna.

The above illustrated approach suffers of some drawbacks. The most important is the limited bandwidth of the conventional micro-strip antennas that introduce distortion and frequency dispersion on the $\mathrm{THz}$ pulse ending with a rapid lengthening of the pulse.
To overcame these limitations electro-optic devices, realised with a non-absorbing material, are used. The interaction mechanism is now a second order process: the rapidly oscillating electric field of the laser pulse excite in the medium a polarisation vector which is proportional to the modulus of the electric field itself. This polarisation vector is the source for an Hertzian vector potential that generates a radiation field. This is a rectification process because the rapid oscillation of the electric field of the laser pulse are compensated in the frequency domain and only the envelope of this pulse remains. The important feature of this device is that, being the medium non absorbing, the polarisation is very fast in following the laser envelope; this means that there is no physical limit in the bandwidth of the $\mathrm{THz}$ pulse that can be generated with this device. The only drawback is that being this a second order process, the intensities of the $\mathrm{THz}$ radiation are smaller respect to the previous cases, but it is now well compensated by the more powerful short-pulse laser source now available also commercially.

\section{A Free Electron Device as THz Radiator}

"Flexibility" is the most relevant feature a Free Electron Device (FED) because any component of such a source can, in principle, be designed in order to emphasise a specific characteristics of the generated radiation [2]. Broadband emission is, in fact, ensured by a short interaction region and by avoiding optical resonators, that usually filter frequency components; these precautions, together with the use of a low energy electron beam, allow an easy and compact design of the source. The most significant peculiarity of a FED is the coherence of an electron beam generated by a Radio-Frequency (RF) accelerator. The coherent emission dominates when the electron bunch length is comparable to wavelength of the radiation to be emitted. As a result coherent emission dominates in the $\mathrm{THz}$ range or at longer wavelengths. Another characteristics of RF based FED is the coherence among the bunches. An RF accelerator generates a train of bunches, and if the correlation among bunches is good, the radiation will be emitted at discrete frequencies which are harmonics of the RF. This is exactly what we have measured experimentally in our compact FED in ENEA [3-4]. A further degree of coherence can be exploited when a proper energyphase correlation is introduced in the electron beam, before entering the interaction region, in order to minimise the negative interferences among the single electrons' emissions. Several techniques can be ap- 
plied to get such a correlation, but the final result is a single-pass emission of a broad-band short pulse emission as reported in Fig. 1(result obtained for an electron beam with $\gamma=15, I_{A V}=20 \mathrm{~mA}$ and an undulator of $\lambda_{u}=2.5 \mathrm{~cm}$ period and $K=1.45$ as magnetic parameter).

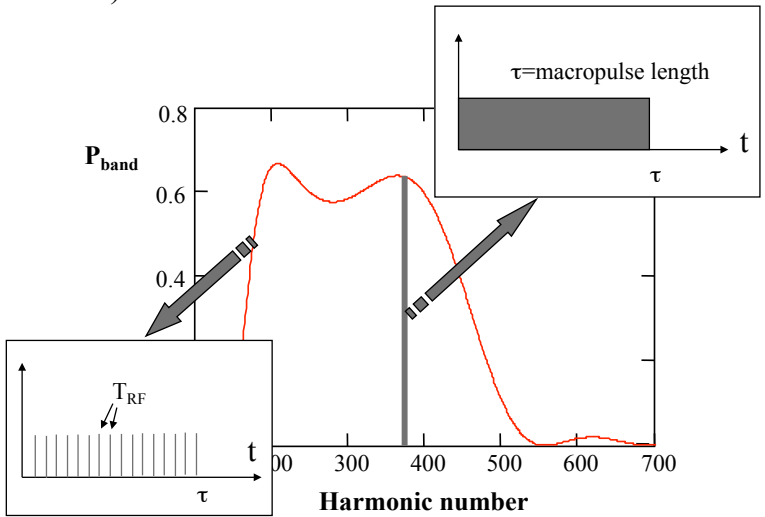

Fig. 1. Power spectrum in a Free Electron Device in the Energy-Phase Correlation regime

Analysing Fig. 1 it is evident that in the EnergyPhase correlation regime it is possible to isolate the single harmonic, with an interferometer, still having an average power for the single frequency around 0.5 W. This is not possible with the conventional $\mathrm{THz}$ sources. Moreover another interesting result is that the single frequency, being an harmonic of the RF has a temporal structure equal to that of the RF macropulse. If we look at the whole bandwidth, the temporal structure is the well known train of microbunches separated by the RF period. In conclusion FED can be considered a convenient flexible and powerful source for the generation of coherent radiation in the $\mathrm{THz}$ spectral region.

A way to get a correlated bunching in the $\mathrm{THz}$ region is the use of a two frequency RF device. Such cavities, in fact, offer appealing possibilities to control the bunch length of an electron beam generated by a RF accelerator. The use of a double frequency cavity requires that the second frequency be an harmonic of the fundamental one. The electrons passing through the cavity will see a field that is the result of the sum of the two fields of the fundamental and its harmonic according to:

$$
V(\phi)=V_{0}\left[\sin \left(\phi+\phi_{S}\right)+k \sin \left(n \phi+\phi_{n}\right)\right]
$$

There are some design parameters that can be set like the harmonic number $n$, the relative amplitude ratio $k$ that contribute to the bunch length, but the most relevant is the relative phase $\left(\phi_{\mathrm{s}}-\phi_{\mathrm{n}}\right)$ between the fundamental and the harmonic. These parameters establish the slope of the total field in the cavity. Two different regimes can be in principle realised: one in which one can realise a bunch-lengthening, which spread can help in damping coherent instabilities that often cut down the bunch lifetime in re-circulated accelerators; and a second in which the particles bunch reduces with respect to the reference electron. In Fig. 2 we report the behaviour of the electron bunch intensity profile as a function of the phase $\phi$, for different harmonics $n$ in the RF cavity $(k=-0.6$, $\left.\phi_{n}=\pi, \phi_{s}=0\right)$.

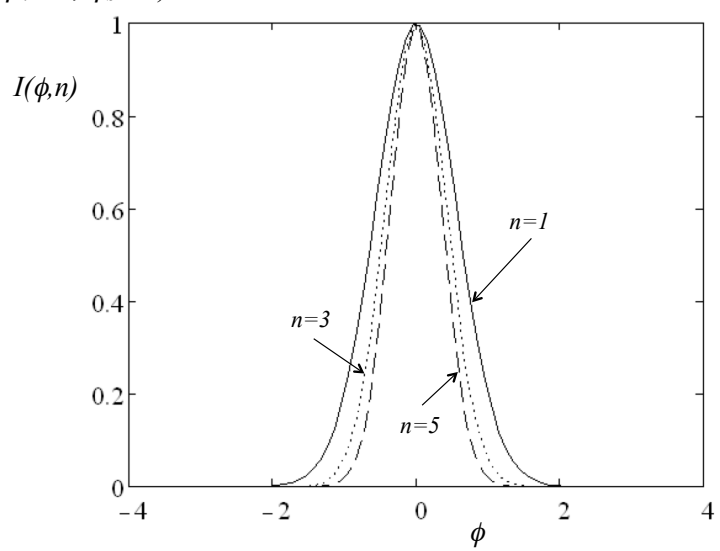

Fig. 2. Bunch shortening in a double frequency RF cavity for different harmonics.

One of the problems suffered by RF accelerator based FED, both normal-conducting and superconducting, is the relatively low efficiency. Considering the basic generation mechanism the power extraction from the electron beam in favour of the radiation never exceed few per cent, both in high gain and low gain regimes. In addition to the use od auto-resonant mechanisms (CARM), a possibility to increase the efficiency of an FED is to design a scheme for recovering the electron beam kinetic energy remained after the interaction. The energy recovered can be used to accelerate a new bunch of electrons thus lowering the accelerator radio-frequency power requirements. The possibility to manage a lower RF power and, at the same time, an exhausted electron beam of low energy, is very useful when designing the device. Generally speaking there are two possibilities for the energy recovering: one is to recycle the electron beam after the interaction region. The second possibility is to recover only the kinetic energy of the electrons before the beam dumps.

Energy recovery devices are particularly useful when designing a radiation source based on superconducting RF accelerators, or on normal conducting cavities working at very low temperatures. These accelerators, in fact, can guarantee a long bunch operation or a quasi-CW operation.

\section{References}

1. Sakai, K. Terahertz Optoelectronics // Berlin: Springer-Verlag. 2005.

2. Dattoli, G., Doria, A., Sabia, E., Artioli, M, Charged Beam Dynamics, Particle Accelerators and Free Electron Lasers // Bristol: IOP Publishing. 2017.

3. Biedron, S., Gallerano, G. P., et al. Compact, High Power Electron Beam based Terahertz Sources // Proc. Of the IEEE 2007. V. 95, No. 8, P. 1666-1678.

4. Doria, A. Relativistic Electron based THz Sources: Principles of Operation and the ENEA Experience // Physics and application of Terahertz Radiation, edited by $\mathrm{M}$. Perenzoni and D. J. Paul. Dordrecht: Springer. 2014. P. 123148. 